\title{
ELECTRICAL AXIS DEVIATION OF FIFTY NORMAL ELECTROCARDIOGRAMS
}

\author{
BY \\ JENNER HOSKIN AND P. JONESCU \\ From the Cardiological Department, Royal Free Hospital \\ Received August 4, 1939
}

In a single muscle fibre the electrical axis-the line along which the greatest electromotive force is developed at a given instant while the muscle is excitedis in the same line as the fibre itself. In a somatic muscle the electrical axis is also in the same line as the muscle itself (Lewis, 1925). In the heart muscle, however, the excitation wave spreads from a normal or abnormal pace-maker and the electrical activity takes a complex path and spreads simultaneously in different directions as the heart fibres run at many different angles.

The diffuse course can be represented by an axis - a curve not lying in a single plane, but in space, as does the heart itself. For clinical use, however, to suit the limits of electrocardiography, the axis is represented by its projection on the anterior plane of the body to fit the Einthoven triangle or the standard leads. Finally the curve is further simplified by constructing the projection of this line on each lead or side of the triangle, and the angle which it makes with one of the sides is briefly used to designate the electrical axis of the electrocardiogram.

The electrical axis can be calculated trigonometrically or by a simple clinical method. In this paper are given the results of measuring the axis deviation in the records from 50 normal female medical students (see Hoskin and Jonescu, pages $33-46$ of this number).

\section{Trigonometrical Method}

The angle of deviation is calculated according to Einthoven's formula. The method is explained very clearly by Lewis $(1925$, p. 108). He points out that unless Einthoven's method is fully appreciated it is impossible to grasp the meaning and significance of these differences in the heights and directions of the deflections as they appear in the three body leads.

The size of angle varies according to the position of the arrow (heart electrical axis) in the triangle and its projection on the leads. Also the angle of the electrical axis varies slightly in relation to a given phase of the cardiac cycle. The direction of the resultant electrical axis of the heart lies within wider limits than does the anatomical axis in normal and abnormal hearts (Paul White, 1937). 
The normal electrical axis lies between the degrees of 0 and 90 . The axis deviations of our 50 normal electrocardiograms are shown in Fig. 1. If the

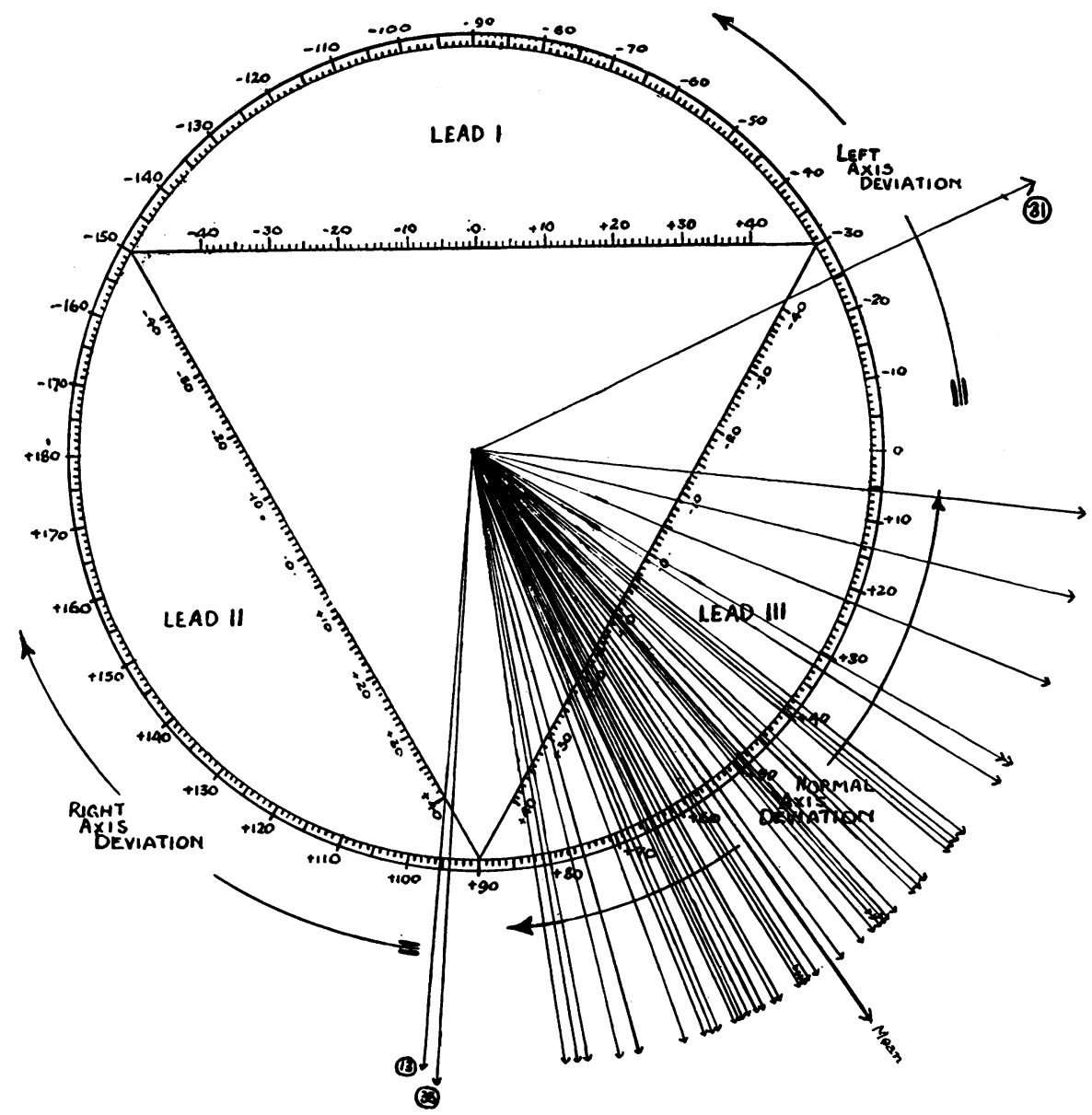

Fig. 1.-Distribution of axis deviation in 50 normal electrocardiograms.

angle has a minus value, that is above the horizontal line, or, in other words, if the arrow (electrical axis) in the Einthoven's triangle has shifted anti-clockwise, there is a so-called left axis deviation. If the angle has a value beyond $90^{\circ}$ and the arrow (electrical axis) in the Einthoven's triangle has shifted clockwise, there is right axis deviation.

The frequency curve constructed from our analysis of the angles of axis deviation is reproduced in Fig. 2. The mean value for the angle of axis deviation was found to be $+54 \cdot 5^{\circ}$. It will be seen from Fig. 1 that in one record from a healthy athletic woman whose heart seemed normal (Case 31) there was an abnormal left axis deviation of $-25^{\circ}$. Two records (Cases 13 and 38) showed a slight right axis deviation $\left(+94^{\circ}\right.$ and $\left.+96^{\circ}\right)$. The rest of the records show an angle within normal limits.

Paul White points out that it is more accurate to use the terms abnormal left and abnormal right axis deviation. Displacement upwards by a high 
diaphragm so that the heart lies horizontally will give left axis deviation, even though the left ventricle remains normal. Similarly, a low diaphragm with a

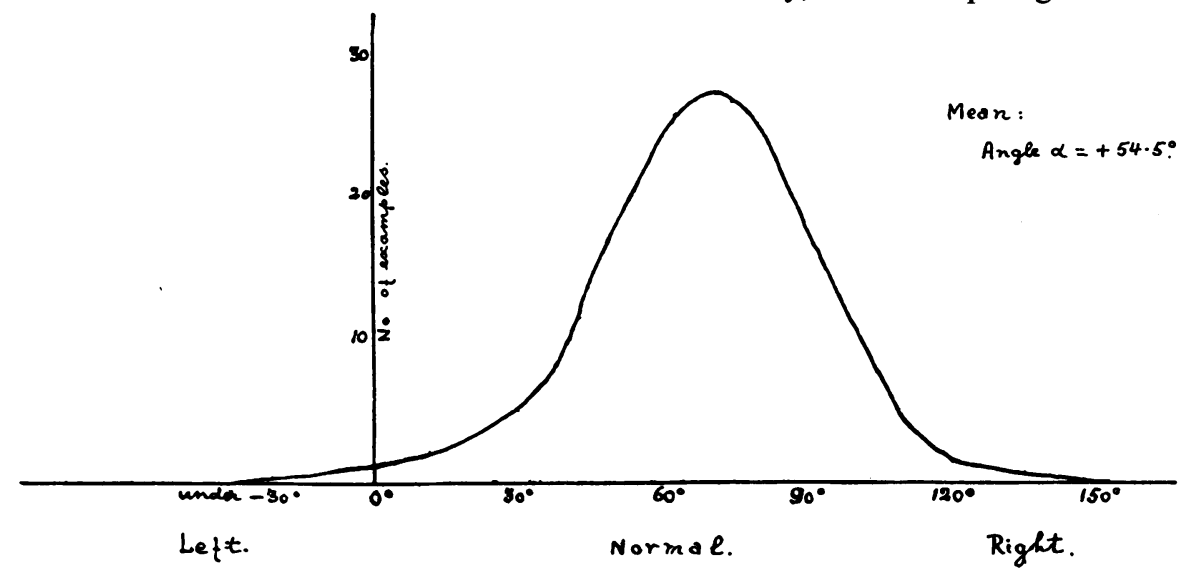

FIG. 2.-Angle of axis deviation : frequency curve.

vertical position of the heart will tend to give an abnormal right axis deviation, even though the right ventricle is small and the left ventricle may be preponderant. It is true, however, that a high degree of abnormal deviation is generally found with enlargement of the right or of the left ventricle ; though serious disease or enlargement of one or the other ventricle may be present with no evident abnormality of axis ; it is also true that when both ventricles are enlarged correspondingly, their effects tend to neutralize each other.

\section{Simple Method. Index of Axis Deviation}

To estimate at a glance in clinical work the axis deviation, without the use of the trigonometrical method, the following formula is used :

$$
\text { Index }=\left(R_{1}+S_{3}\right)-\left(R_{3}+S_{1}\right)
$$

The index is derived by subtracting the sum of the amplitudes in millimetres of $\mathbf{R}$ in lead III and $\mathbf{S}$ in lead I from the sum of the amplitudes of $R$ in lead $I$ and $S$ in lead III. According to Paul White, if the index measures over $30 \mathrm{~mm}$., abnormal left axis deviation is present with a wide borderline of $20-30 \mathrm{~mm}$. If the index measures under $-15 \mathrm{~mm}$., abnormal right axis deviation is present with a normal borderline of -10 to $-15 \mathrm{~mm}$. The analysis of index of axis deviation in our 50 normal subjects is reproduced in the following table :

Index of Axis Deviation in 50 Normal Cases

\begin{tabular}{|c|c|c|}
\hline & Range of Index in $\mathrm{mm}$. & Percentage of Total \\
\hline $\begin{array}{l}\text { Right } \\
\text { Left }\end{array}$ & 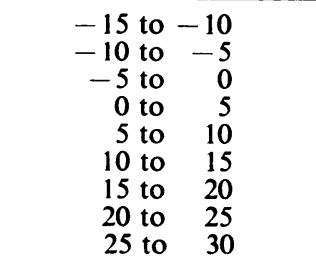 & $\begin{array}{r}4 \\
10 \\
28 \\
24 \\
20 \\
6 \\
6 \\
6 \\
0 \\
2\end{array}$ \\
\hline
\end{tabular}


The mean value was $+2.45 \mathrm{~mm}$. One record (Case 31) showed left axis deviation that was almost abnormal $(25-30 \mathrm{~mm}$.) and three records (Cases 20,21 , and 45) showed slight or borderline left axis deviation (15-20 mm.). Two records (Cases 43 and 49) showed slight or borderline right axis deviation. The examination of the table and curves shows that in general both methodsthe trigonometrical and the simple-agree, but that the trigonometrical is much more accurate. It will be seen that one electrocardiogram (Case 13) is shown by the trigonometrical method to be of right axis deviation, but by the simple method is found to be within normal range (index $=-8$ ). Also that the 5 records ( 2 of which were borderline right axis and 3 borderline left axis) are found to belong to the normal range of electrical axis deviation by the trigonometrical method.

\section{SUMMARY}

The results of the measurements of the axis deviation of the electrocardiograms taken from 50 normal women students at the Royal Free Hospital have been measured and reported.

A comparison of the trigonometrical method and of a simple clinical method of calculation shows that there is a general agreement. The former is, of course, more accurate but the latter can be calculated quickly and easily and is accurate enough to be of practical use.

We wish to express our thanks to Professor Winifred Cullis and Miss Marion Morris from the London School of Medicine for Women for their valuable help and advice and to the students of the Royal Free Hospital for their kind collaboration.

\section{REFERENCES}

Lewis, T. (1925). The Mechanism and Graphic Registration of the Heart Beat (3rd ed.). London.

White, P. D. (1937). Heart Disease. New York. 